\title{
Reconstruction of a large-scale reconnection exhaust structure in the solar wind
}

\author{
W.-L. Teh ${ }^{1}$, B. U. Ö. Sonnerup ${ }^{1}$, Q. Hu ${ }^{2}$, and C. J. Farrugia ${ }^{3}$ \\ ${ }^{1}$ Thayer School of Engineering, Dartmouth College, NH, USA \\ ${ }^{2}$ Institute of Geophysics and Planetary Physics, UCR, CA, USA \\ ${ }^{3}$ Institute for the Study of Earth, Ocean, and Space, UNH, NH, USA
}

Received: 28 August 2008 - Revised: 22 December 2008 - Accepted: 22 January 2009 - Published: 18 February 2009

\begin{abstract}
We recover two-dimensional (2-D) magnetic field and flow field configurations from three spacecraft encounters with a single large-scale reconnection exhaust structure in the solar wind, using a new reconstruction method (Sonnerup and Teh, 2008) based on the ideal single-fluid MHD equations in a steady-state, 2-D geometry. The reconstruction is performed in the rest frame of the X-line, where the flow into, and the plasma jetting within, the exhaust region are clearly visible. The event was first identified by Phan et al. (2006) in the ACE, Cluster, and Wind data sets; they argued that quasi-steady reconnection persisted for over $2 \mathrm{~h}$ at a long $\left(390 R_{E}\right) \mathrm{X}$-line. The reconnection exhaust is sandwiched between two discontinuities, both of which contain elements of intermediate- and slow-mode behavior; these elements are co-located rather than being spatially separated. These composite discontinuities do not satisfy the coplanarity condition or the standard MHD jump conditions. For all three spacecraft, the Walén regression line slope was positive (negative) for the leading (trailing) discontinuity. Our MHD reconstruction shows that: (1) the X-line orientation was close to the bisector of the overall magnetic shear angle and exhibited a slow rotating motion toward the Sun-Earth line; (2) the X-line moved earthward, dawnward, and southward; (3) the reconnection electric field was small $(\sim 0.02 \mathrm{mV} / \mathrm{m}$ on average) and gradually decreased from the first crossing (ACE) to the last (Wind). The magnetic field and flow field configurations recovered from ACE and Cluster are similar while those recovered from Wind also include a magnetic island and an associated vortex. Reconnection persisted for at least $2.4 \mathrm{~h}$ involving inflow into the exhaust region from its two sides. Time-dependence in the reconnection electric fields seen by ACE and Wind indicates local
\end{abstract}

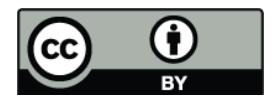

Correspondence to: W.-L. Teh (wai-leong.teh@lasp.colorado.edu) temporal variations in the field configuration. In addition to the reconstruction results, we provide a description and analysis of many details from the crossings by the spacecraft.

Keywords. Space plasma physics (Discontinuities; Kinetic and MHD theory; Magnetic reconnection)

\section{Introduction}

Magnetic reconnection is an important and efficient process for converting magnetic field energy to plasma kinetic and thermal energy. In this process, the magnetic field topology is changed by breaking and reconnecting oppositely directed frozen-in field lines from different plasma regions and the plasma flow is accelerated to speeds comparable to the Alfvén speed. Reconnection plays a crucial role in highenergy processes in the solar corona. It is also essential for the entry of solar-wind plasma into the magnetosphere from the magnetosheath, and in accounting for magnetic substorms in the geomagnetic tail. In the past decade, many detailed in-situ observations have been reported as evidence for the occurrence of reconnection at the magnetopause, in the geomagnetic tail, and, recently, in the magnetosheath and in the solar wind.

Magneto-hydrostatic Grad-Shafranov (GS) reconstruction was originally proposed by Sonnerup and Guo (1996) and then further developed by Hau and Sonnerup (1999) and Hu and Sonnerup (2003). It is a method of solving the GS equation as a spatial initial-value problem to produce maps of 2$\mathrm{D}$, time-independent field and plasma structures, from data taken by one or more spacecraft traversing the structures. Later, a general GS-like reconstruction theory was developed (Sonnerup et al., 2006), which included the presence of field-aligned flows and also, as a separate case, flow transverse to a unidirectional magnetic field. Recently, Sonnerup

Published by Copernicus Publications on behalf of the European Geosciences Union. 


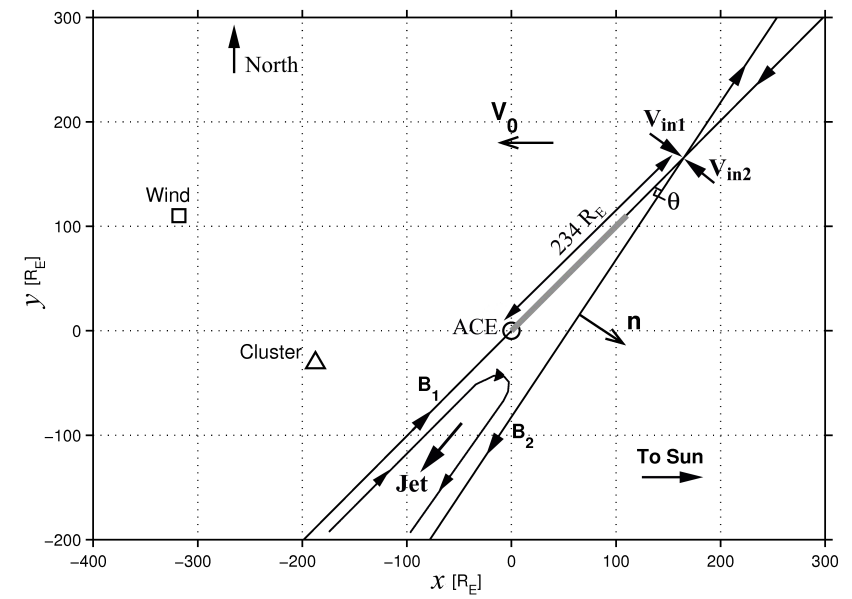

Fig. 1. Sketch of the encounters of ACE (circle), Cluster (triangle), and Wind (square) with reconnection exhaust in the solar wind, on 2 February, 2002, in the reconstruction plane, i.e. perpendicular to the $\mathrm{X}$-line orientation (the $\hat{z}$ axis). The $\hat{\boldsymbol{x}}$ axis is along the vector $-\boldsymbol{V}_{0} /\left|\boldsymbol{V}_{0}\right|$, representing the X-line motion; the axis $\hat{\boldsymbol{y}}=\hat{\boldsymbol{z}} \times \hat{\boldsymbol{x}}$, is directed approximately along the $+\hat{\boldsymbol{z}}_{\mathrm{GSE}}$ axis. The axes $\hat{z}=[+0.428-0.832-0.353]$ and $\hat{\boldsymbol{x}}=[+0.905+0.387+0.178]$ used in the figure are obtained by averaging the $\mathrm{X}$-line orientations and its motions over the three spacecraft. The gray bar denotes the lower bound of $156 R_{E}$ for the distance between the X-line and the ACE spacecraft at the time of the ACE encounter with the reconnection exhaust. This lower bound is based on the assumption that the X-line itself was encountered by Wind. In reality, the X-line was at a substantially larger $y$-value, i.e. it was substantially north of Wind.

and Teh (2008) have developed a new reconstruction method, based on the ideal single-fluid MHD equations in a steadystate, 2-D geometry. This so-called MHD reconstruction is no longer based on a GS-like equation and, for reconnection events, can be performed in the frame of reference moving with the X-line rather than in the deHoffmann-Teller (HT) frame used in GS reconstruction, in which the flow is fieldaligned. Subsequently, Teh and Sonnerup (2008) applied the MHD reconstruction method to a reconnection event at the magnetopause that had been previously reconstructed in the magneto-hydrostatic approximation (Hasegawa et al., 2004, 2005), and also by including field-aligned flow (Teh et al., 2007). The new reconstruction results showed the reconnection geometry in unprecedented detail, including 2-D maps of magnetic field and flow field, as well as electric current and electric potential distributions. The results indicated the presence of an X-line in the magnetopause and the inflow into, and acceleration of plasma within, a wedge of reconnected field lines.

Recently, reconnection events have been found to occur in the solar wind and have been documented extensively (e.g. Gosling et al., 2005; Phan et al., 2006; Gosling et al., 2007). These observations indicate that reconnection in solar-wind current sheets is a common occurrence that continues for hours, probably at variable reconnection rates, and is associated with an extended X-line. The reconnection jet or exhaust in these events is confined within a bifurcated current sheet akin to a Petschek-type exhaust, i.e. the plasma jet is bounded on its two sides by a combination of a rotational discontinuity and a slow-mode shock (Petschek and Thorne, 1967).

Reconnection events in the solar wind have large spatial scales and therefore are good candidates for use of the MHD reconstruction method. In this paper, we apply the method to an event, first identified by Phan et al. (2006), that occurred in the solar wind on 2 February 2002. It was observed by widely separated spacecraft (ACE, Cluster, and Wind) in sequence, so as to establish its enormous size and long duration.

The paper is organized as follows. Section 2 contains an overview of the observations and shows a number of analysis results for each spacecraft: Orientation and motion of the current sheet; magnetic field hodograms and Walén tests; Xline orientation and motion; and reconnection electric fields. In Sect. 3, the mathematical basis for the reconstruction is briefly summarized and reconstruction results from each of the three spacecraft are presented. A summary and discussion is provided in Sect. 4.

\section{Overview of observations}

On 2 February 2002, the ACE and Cluster spacecraft were at GSE coordinates $[236,-33,23] R_{E}$ and $[14,10,5] R_{E}$, respectively, while the Wind spacecraft was near apogee at $[9,-321,16] R_{E}$. They formed a huge triangular configuration in the solar wind, compared to which the Cluster spacecraft separation of less than $1000 \mathrm{~km}$ is negligibly small. For this reason, we sometimes refer to the four Cluster spacecraft, $\mathrm{C} 1-\mathrm{C} 4$, as if they were a single spacecraft. Figure 1 shows a schematic of the spacecraft locations and the encounters with the reconnection exhaust in the plane perpendicular to the X-line orientation. The earlier study of the event by Phan et al. (2006) showed that a reconnection Xline must have existed north of all spacecraft, as illustrated in Fig. 1, and must have had a length of at least $390 R_{E}$. These authors also argued that the reconnection was in a quasi-steady state for over $2.5 \mathrm{~h}$.

The matrix of panels in Fig. 2 shows the magnitude and GSE components of the magnetic field (1st row) and the ion velocity (2nd row), the ion density and temperature (3rd row), as well as the ion pressure, magnetic pressure, and their sum (4th row) for ACE (1st column), Cluster (2nd column), and Wind (3rd column). The magnetic field and plasma data are from the MAG and SWEPAM instruments onboard ACE, the FGM and CIS/HIA instruments onboard Cluster, and the MFI and 3DP instruments onboard Wind, with time resolution $64 \mathrm{~s}, 4 \mathrm{~s}$, and $3 \mathrm{~s}$, respectively. The data are taken from the CDAWeb and the Cluster Active Archive (CAA) website. 

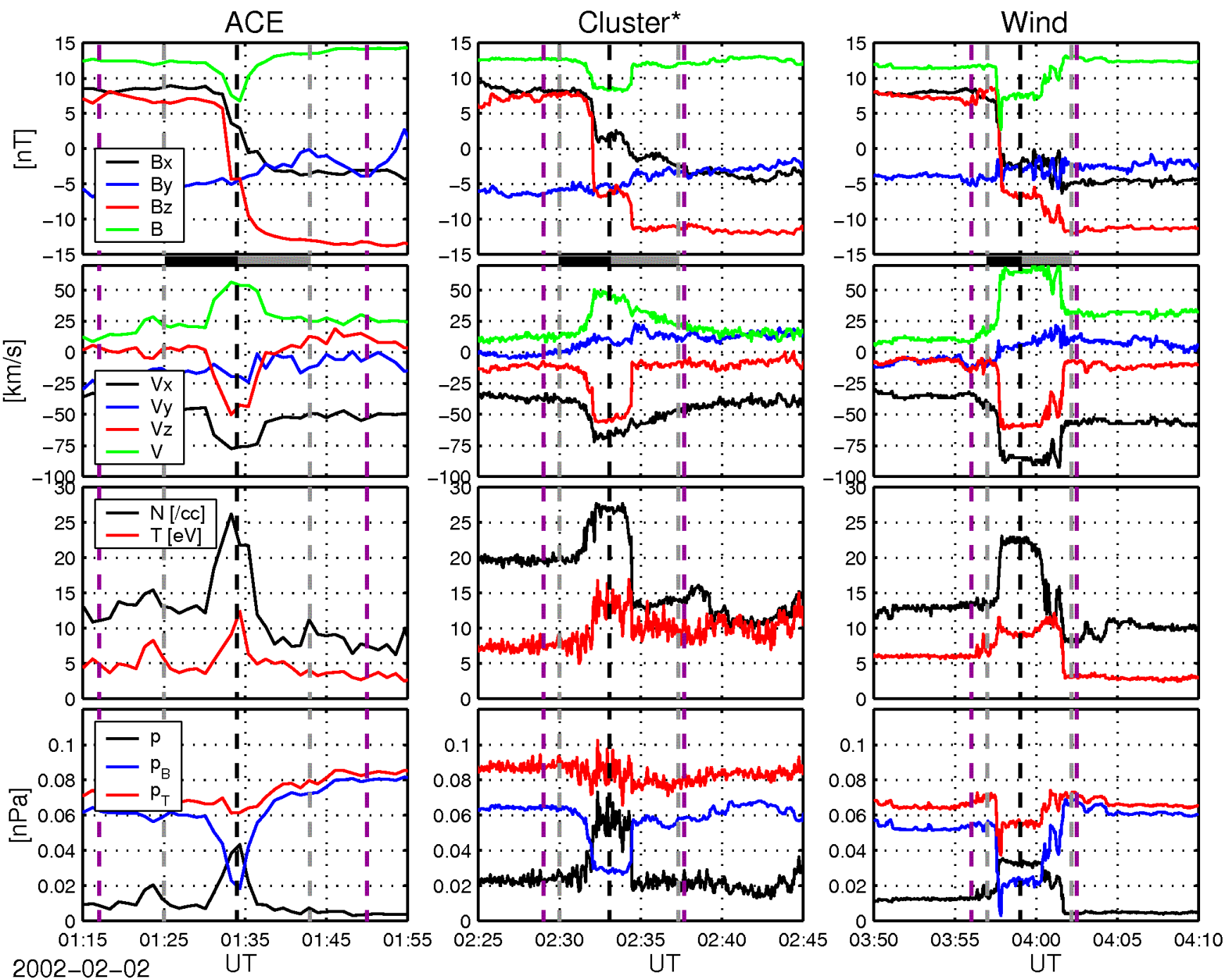

$\mathrm{V} x=\mathrm{V} x+300, \mathrm{~V}=\mathrm{V}-325$, vector in GSE

Fig. 2. Time series measurements of ACE, Cluster, and Wind in the solar wind on 2 February 2002. The panels in row, from top to bottom, are the magnetic field (GSE), the ion plasma velocity (GSE), the ion density and temperature, and the ion pressure $p$, the magnetic pressure $p_{B}$, as well as the total pressure $\left(p_{T}=p+p_{B}\right)$, respectively. The Cluster data is combined from C1 and C3. The black (gray) bar denotes the interval for leading (trailing) discontinuity. The two purple (dashed gray) lines denote the interval for the reconstruction domain (the bifurcated current sheet).

The Cluster data are combined from $\mathrm{C} 1$ and $\mathrm{C} 3$; the temperature shown for Cluster is $T_{\perp}$, because the measured $T_{\|}$values do not appear reliable during the event. The magnetic field measurements for $\mathrm{C} 2$ and $\mathrm{C} 4$ (not shown) are similar to those from $\mathrm{C} 1$ and $\mathrm{C} 3$. A reconnection jet, referred to as a region of reconnection exhaust, was identified by Phan et al. (2006) in the data from ACE, Cluster, and Wind. It is characterized by enhanced plasma speed, density, temperature, and thermal pressure, accompanied by a lower magnetic pressure. It is bounded on its two sides by discontinuities, i.e. it is in effect embedded in a bifurcated current sheet. As found by Phan et al. (2006), Fig. 2 shows that the accelerated plasma flow within the reconnection exhaust pointed southward (negative $V_{z \mathrm{GSE}}$ ) and earthward (negative $V_{x \mathrm{GSE}}$ ) for all three spacecraft, which is consistent with the presence of an X-line at a location well north of the ecliptic plane as illustrated in Fig. 1.

\subsection{Orientation and motion}

In the 2 February 2002 event, the bifurcated current sheet was observed, first by ACE near the L1 point at $\sim 01: 34$ UT, then by Cluster upstream of the Earth's bow shock about $1 \mathrm{~h}$ later, and finally by Wind dawnward of the Cluster location and about $2.42 \mathrm{~h}$ after ACE. Using the relative position vectors 
Table 1. Summary of MVAB results for ACE, Cluster and Wind.

\begin{tabular}{lllllcccr}
\hline & Time interval [UT] & \multicolumn{3}{c}{$\hat{\boldsymbol{n}}^{\mathrm{MVAB}}[\mathrm{GSE}]$} & $\lambda_{2} / \lambda_{3}$ & $B_{n}[\mathrm{nT}]$ & $\theta^{\mathrm{a}}$ & $\phi^{\mathrm{b}}$ \\
\hline \multirow{4}{*}{ ACE } & $01: 25: 00-01: 34: 00^{\mathrm{c}}$ & 0.785 & 0.513 & -0.348 & 10.4 & +1.8 & & \\
& $01: 34: 00-01: 43: 00^{\mathrm{d}}$ & 0.806 & 0.352 & -0.477 & 5.2 & +3.0 & & \\
& $01: 25: 00-01: 43: 00^{\mathrm{e}}$ & 0.779 & 0.498 & -0.381 & 9.2 & +1.6 & & 4.9 \\
\hline \multirow{4}{*}{ Cluster** $^{*}$} & $02: 30: 00-02: 33: 05^{\mathrm{c}}$ & 0.459 & 0.867 & -0.191 & 1.7 & -2.6 & & \\
& $02: 33: 05-02: 37: 20^{\mathrm{d}}$ & 0.391 & 0.912 & 0.126 & 2.7 & -5.2 & & \\
& $02: 30: 00-02: 37: 20^{\mathrm{e}}$ & 0.780 & 0.526 & -0.339 & 3.4 & +0.7 & & 3.2 \\
\hline \multirow{4}{*}{ Wind } & $03: 57: 00-03: 59: 00^{\mathrm{c}}$ & 0.640 & 0.699 & -0.319 & 4.4 & -1.4 & \multirow{2}{*}{4.7} & \\
& $03: 59: 00-04: 02: 10^{\mathrm{d}}$ & 0.655 & 0.651 & -0.383 & 14.4 & -0.4 & & \\
& $03: 57: 00-04: 02: 10^{\mathrm{e}}$ & 0.648 & 0.697 & -0.308 & 9.5 & -1.2 & & 9.5 \\
& $03: 57: 00-04: 02: 10^{\mathrm{f}}$ & 0.659 & 0.610 & -0.441 & & -0.25 & & 7.7 \\
\hline
\end{tabular}

*Combined data from $\mathrm{C} 1$ and $\mathrm{C} 3$. $^{\mathrm{a}}$ The angle between the normals of the leading and trailing discontinuities. $\mathrm{b}$ The angle between the global normal and that of the combined bifurcated structure. ${ }^{\mathrm{c}}$ Leading discontinuity. ${ }^{\mathrm{d}}$ Trailing discontinuity. ${ }^{\mathrm{e}}$ Combined bifurcated current sheet. ${ }^{\mathrm{f}}$ The adjusted normal.

Table 2. Summary of $\boldsymbol{V}_{\mathrm{HT}}$ and $V_{n}$ results for ACE, Cluster and Wind.

\begin{tabular}{lccccc}
\hline & Time interval [UT] & \multicolumn{3}{c}{$V_{\text {HT }}[\mathrm{GSE}][\mathrm{km} / \mathrm{s}]$} & $V_{n}[\mathrm{~km} / \mathrm{s}]$ \\
\hline \multirow{4}{*}{ ACE } & $01: 25: 00-01: 34: 00$ & -388.1 & 6.5 & -32.5 & -289.9 \\
& $01: 34: 00-01: 43: 00$ & -368.8 & -16.6 & -52.3 & -278.0 \\
& $01: 25: 00-01: 43: 00$ & -364.2 & -8.7 & -18.3 & $-280.9 /-278.5^{\mathrm{a}}$ \\
\hline \multirow{3}{*}{ Cluster* } & $02: 30: 00-02: 33: 05$ & -370.4 & 23.4 & -41.6 & -141.9 \\
& $02: 33: 05-02: 37: 20$ & -360.0 & -0.9 & -61.4 & -149.1 \\
& $02: 30: 00-02: 37: 20$ & -359.7 & 12.8 & -34.9 & -262.0 \\
\multirow{3}{*}{ Wind } & $03: 57: 00-03: 59: 00$ & -377.4 & 11.2 & -46.7 & -218.7 \\
& $03: 59: 00-04: 02: 10$ & -392.3 & -0.1 & -81.3 & -226.5 \\
& $03: 57: 00-04: 02: 10$ & -382.6 & 9.0 & -54.0 & $-224.8 /-222.7^{\mathrm{b}}$ \\
\hline
\end{tabular}

*Combined data from $\mathrm{C} 1$ and $\mathrm{C} 3$. a Adjusted speed for the constant axial electric field. b Adjusted speed when $\hat{\boldsymbol{n}}=[+0.659,+0.610,-0.441]$.

and the propagation times of the current sheet from ACE to Cluster and from ACE to Wind, we can estimate the global orientation of the vector $\hat{\boldsymbol{n}}$ normal to the current sheet and its average speed. In general, such a determination requires four spacecraft (e.g. Harvey, 1998). If only three are available, the average orientation and speed can still be obtained by use of the constraint that $\hat{\boldsymbol{n}}$ be perpendicular to the maximum variance direction for the combined set of magnetic field vectors from the three spacecraft. Details of the method are given in the Appendix. The resulting global normal vector of the current sheet is $\hat{\boldsymbol{n}}=[+0.745,+0.570,-0.347](\mathrm{GSE})$, chosen to point sunward. It forms an angle of only $3.6^{\circ}$ with the normal estimated by Phan et al. (2006) using minimum variance analysis of the magnetic field (MVAB) (Sonnerup and Cahill, 1967) from Wind. The average speed of the current sheet along the global normal direction was found to be $V_{n}=-242.6 \mathrm{~km} / \mathrm{s}$ (anti-sunward). Table 1 summarizes the analysis results from MVAB for each spacecraft, including the normal $\hat{\boldsymbol{n}}$, the ratio of intermediate to minimum eigenvalue $\left(\lambda_{2} / \lambda_{3}\right)$ from MVAB, the average magnetic normal component $B_{n}$, and the two angles, $\theta$ and $\phi$. Here $\theta$ is the wedge angle between the normals of the leading and trailing discontinuities (see Fig. 1) and $\phi$ is the angle between the global normal given above and that obtained from MVAB of the combined bifurcated current sheet. The ACE and Wind intervals give MVAB eigenvalue ratios ranging from marginal $\left(\lambda_{2} / \lambda_{3}=4 \sim 5\right)$ to good $\left(\lambda_{2} / \lambda_{3}>9\right)$; the result indicates that the angle $\theta$ is consistent with an X-line north of the spacecraft and that it is larger for ACE than for Wind. The poor eigenvalue ratio $\left(\lambda_{2} / \lambda_{3}<4\right)$ for Cluster means that the corresponding large value of $\theta$ probably is not meaningful. The results for the angle $\phi$ indicate that the normal of the combined bifurcated current sheet is within $10^{\circ}$ of the global normal given above for all three spacecraft. 
We note that the number of decimal places of the various vector components, velocities, and angles, given above and elsewhere in the paper, do not represent actual accuracy; they are provided to permit precise verification of our calculations.

Table 2 summarizes the results of the deHoffmann-Teller (HT) frame analysis, including the discontinuity speed $V_{n}=\boldsymbol{V}_{\mathrm{HT}} \cdot \hat{\boldsymbol{n}}$, where $\boldsymbol{V}_{\mathrm{HT}}$ is the HT frame velocity relative to the spacecraft. As expected in the solar wind, all three crossings have good HT frames with the main component of the frame velocity directed along $-\hat{\boldsymbol{x}}_{\mathrm{GSE}}$. The $V_{n}$ values for the combined bifurcated structure indicate that the motion of the current sheet was slowing down from ACE $(-278.5 \mathrm{~km} / \mathrm{s})$ to Cluster $(-262.0 \mathrm{~km} / \mathrm{s})$ and then to Wind $(-222.7 \mathrm{~km} / \mathrm{s})$. As shown in the table, we have made a small adjustment of the $V_{n}$ values for ACE and Wind, for reasons to be discussed for ACE in Sect. 2.3 and for Wind below. Their average, $-254.4 \mathrm{~km} / \mathrm{s}$, is reasonably close to the speed of $-242.6 \mathrm{~km} / \mathrm{s}$, given above for the global calculation. Since the $\mathrm{X}$-line location for all three crossings is north of the ecliptic plane, i.e. north of all three spacecraft, and since the GSE $B_{z}$ component (top row in Fig. 2) changes from positive to negative, the field component $B_{n}$ along the sunward pointing normal of the current sheet should be positive, as illustrated in Fig. 1. To meet this condition, the Wind normal for the combined bifurcated current sheet must be adjusted. We did this by rotating the normal vector, estimated from MVAB with the constraint of $\hat{\boldsymbol{n}} \cdot \hat{\boldsymbol{z}}=0$, about the invariant axis $\hat{z}$ by an angle of $6^{\circ}$. The invariant axis, $\hat{z}=[+0.471,-0.791,-0.391]$ (GSE), we use for Wind is the one chosen by Phan et al. (2006) as the bisector of angle between the fields on the two sides of the bifurcated current sheet. The resulting normal $\hat{\boldsymbol{n}}$ leads to a positive $B_{n}$ of $+0.2 \mathrm{nT}$ for the trailing discontinuity, but the $B_{n}$ of the leading discontinuity still remains negative, at $-1.0 \mathrm{nT}$; the resulting adjusted $V_{n}$ value for the combined bifurcated structure is $-222.7 \mathrm{~km} / \mathrm{s}$, which is only a small deviation from the previous result of $\boldsymbol{V}_{\mathrm{HT}} \cdot \hat{\boldsymbol{n}}=-224.8 \mathrm{~km} / \mathrm{s}$ (see Table 2). The reason for the negative $B_{n}$ value remaining in the leading discontinuity seen by Wind will become clear from the MHD reconstruction results. For the Cluster encounter, the negative $B_{n}$ values for the two individual discontinuities are due to the poor normal vector estimation, but the normal for the combined current sheet, which is within $3.2^{\circ}$ of the global normal orientation, gives positive $B_{n}$ for both discontinuities.

\subsection{Magnetic field hodograms and Walén test}

Figure 3 shows the magnetic field hodogram on the plane perpendicular to the normal vector for each spacecraft and each discontinuity. Note that the $\mathrm{x}-(\mathrm{y}-)$ axis in the hodogram is along the intermediate (maximum) variance direction of the magnetic field, and the normal vector is obtained from MVAB for each separate discontinuity, except that the Cluster normal is the same for both discontinuities and is esti- mated from the entire data interval of the bifurcated structure; this is done because of the poor eigenvalue ratio for each individual discontinuity. The angle between the two dashed lines in Fig. 3 represents the magnetic shear angle between the leading and trailing edges of the discontinuity. We find that the magnetic shear angle for the leading (trailing) discontinuity is gradually increasing (decreasing) from $70^{\circ}$ $\left(65^{\circ}\right)$ for ACE, to $90^{\circ}\left(38^{\circ}\right)$ for Cluster, and finally to $137^{\circ}$ $\left(10^{\circ}\right)$ for Wind. Thus the total shear angle across the bifurcated current sheet first decreases from $135^{\circ}$ (ACE) to $128^{\circ}$ (Cluster), and then increases to $147^{\circ}$ (Wind). In the MHD description (Petschek and Thorne, 1967), each discontinuity should consist of two parts. First is a leading rotational discontinuity (intermediate-mode wave), in which any field rotation needed takes place, without change in field magnitude or plasma properties. It is followed by a slow-mode shock, in which no net field rotation occurs but the field magnitude decreases and the plasma pressure, density, temperature, and entropy all increase. However, the hodograms do not show any clear spatial separation between intermediateand slow-mode behaviors: for unknown reasons, both seem to occur together even though the distance to the reconnection site is large (see Sect. 4). The overall field change in the trailing (sunward-facing) discontinuity seen by Wind is close to that expected across a slow-mode shock in that the field rotation across it is only $10^{\circ}$, i.e. the coplanarity condition is nearly satisfied. But, the details of the field behavior in the discontinuity are not readily accounted for. The Wind hodogram for the leading (earthward-facing) discontinuity is not an MHD rotational discontinuity because the field magnitude decreases across it. It is akin to an intermediate MHD shock, except that the field rotation is not $180^{\circ}$ but only $137^{\circ}$. The other MHD jump conditions are also not satisfied. The discontinuities seen by the other spacecraft show even stronger deviations from MHD predictions.

Figure 3 also illustrates that the component of the tangential field that is perpendicular to the X-line (the $\hat{z}$-axis, denoted by an arrow) reverses in the leading discontinuity (top panels) but not in the trailing one (bottom panels).

Figure 4 shows the Walén plot, i.e. a plot of the components of $\left(\boldsymbol{V}-\boldsymbol{V}_{\mathrm{HT}}\right)$ versus the corresponding components of the Alfvén velocity $\boldsymbol{V}_{A}=\boldsymbol{B} / \sqrt{\mu_{0} \rho}$, for each spacecraft and each discontinuity. Components along the natural coordinates of each event $\left(\hat{\boldsymbol{x}^{\prime}}=\hat{\boldsymbol{n}} \times \hat{\boldsymbol{z}}, \hat{\boldsymbol{y}^{\prime}}=\hat{\boldsymbol{n}}, \hat{\boldsymbol{z}}^{\prime}=\hat{\boldsymbol{z}}\right)$, where $\hat{z}$ is the invariant axis, are used in the plots. The first (second) row in Fig. 4 is the Walén plot for the leading (trailing) discontinuity for each spacecraft. The normal $\hat{\boldsymbol{n}}$ is obtained from MVAB for the combined bifurcated structure, i.e. from data within the region enclosed by the two vertical dashed gray lines in Fig. 2; the choice of the invariant axis $\hat{z}$ will be discussed in the next section. The adjusted normal is used in the two Walén plots for Wind. Although the slope of the Walén regression line is only very weakly dependent on the coordinate system used, the components along the natural 

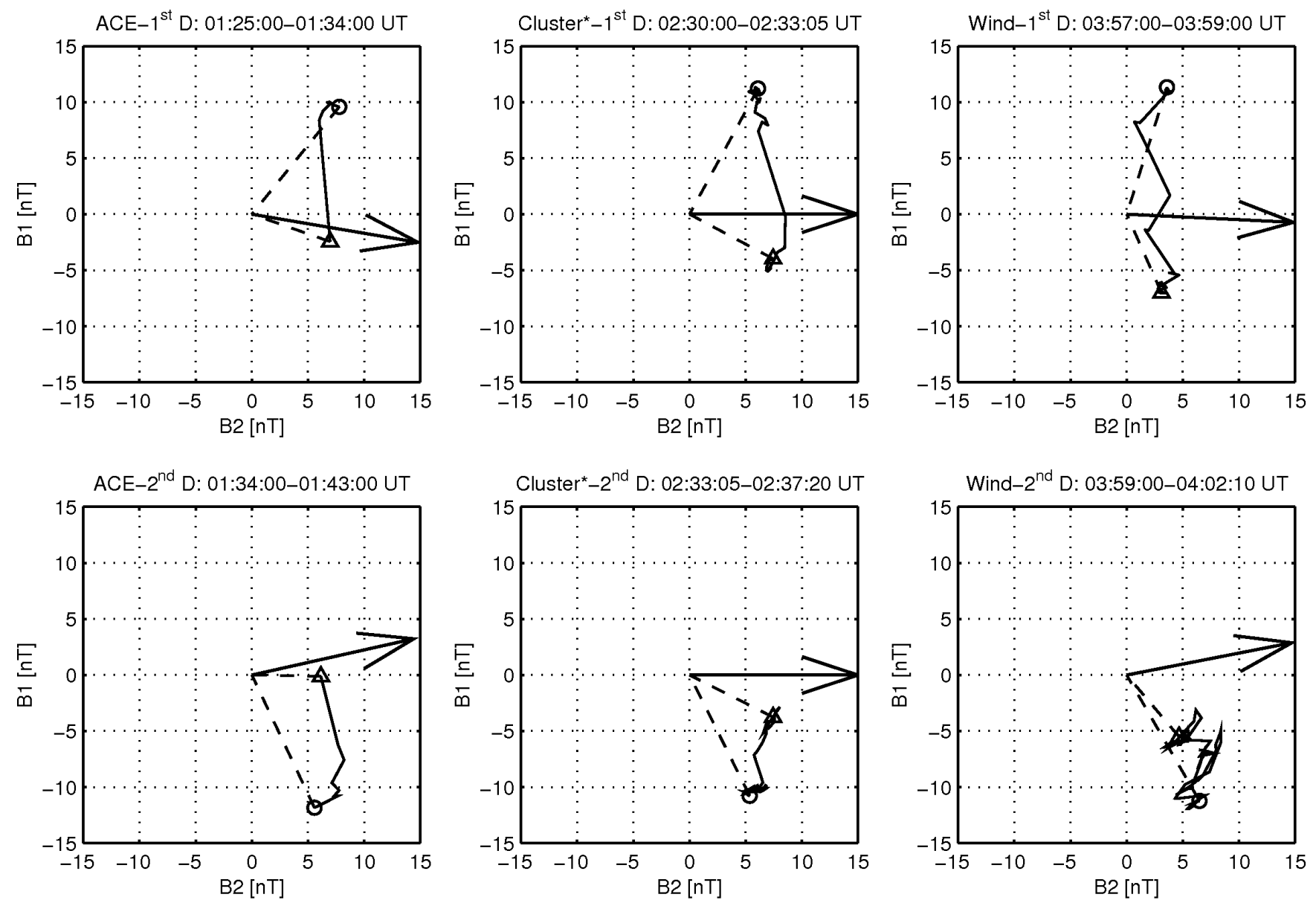

Fig. 3. Magnetic-field hodograms for each spacecraft and each discontinuity. The first (second) row is for leading (trailing) discontinuity; the circle (triangle) denotes the starting (ending) point. The field component B1 (B2) is the magnetic field projected on the maximum (intermediate) variance direction from MVAB. The angle between the two dashed lines represents the magnetic shear angle across the leading and trailing edges of the exhaust region. Arrows are the invariant axis, projected onto the plane.

coordinate axes help illustrate the small flow (blue triangles) along the normal vector as well as the reversal of the tangential field and flow in the leading discontinuity and the absence of such reversal in the trailing discontinuity. We see that all three spacecraft observed a positive (negative) Walén regression line slope for the leading (trailing) discontinuity, as expected on the two sides of the exhaust wedge. The average Walén slope is $\sim \pm 0.7$, indicating that the plasma flow speeds, when seen in the HT frame, are $\sim 70 \%$ of the Alfvén speed in the direction parallel or antiparallel to the magnetic field. Since pressure anisotropy and the pressure of $\alpha$ particles are not taken into account in our determination of the Alfvén speed, the actual Walén slope may be somewhat different. In the HT frame, we find, as expected, that ACE and Cluster have a small positive (negative) average value of the plasma flow along the normal $\hat{\boldsymbol{n}}$ for the leading (trailing) discontinuity; there is an exception to this behavior for the leading (earthward-facing) discontinuity seen by Wind, which has a negative average value of this flow. For ACE and
Cluster, the positive (negative) flow values along the normal vector, shown by the blue triangles in the Walén plots, indicate that there is inflow into the exhaust region from the adjoining solar wind across both the earthward-facing and the sunward-facing discontinuities, as illustrated in Fig. 1. In Sect. 3.1, we will discuss the exception encountered in the Wind event by use of the MHD reconstruction results.

\subsection{X-line orientation and motion, and reconnection electric field}

For time independent, two-dimensional structures, the axial electric field $E_{z}$ is constant, as a consequence of Faraday's law. Accordingly, the $\mathrm{X}$-line orientation, $\hat{z}$, and its motion, $\boldsymbol{V}_{0}$, can in principle be obtained from the method by Sonnerup and Hasegawa (2005). However, this method is sensitive to time variations and fails to give believable results for our events, where such variations are in clear evidence (see discussion below and also in Sect. 4). For multi-spacecraft 

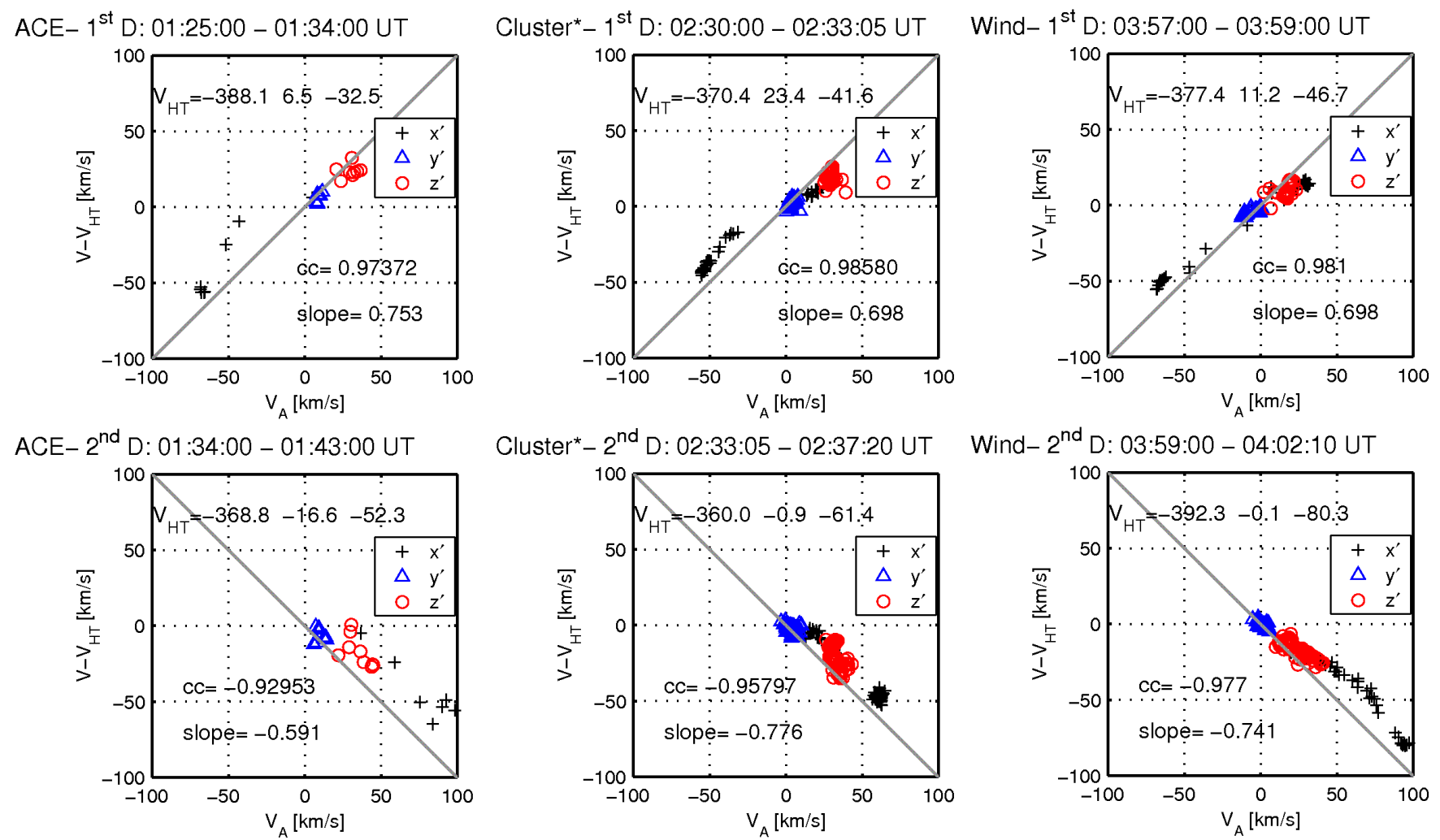

Fig. 4. Walén plots of the leading discontinuity (1st D) and the trailing discontinuity (2nd D) for ACE (1st column), Cluster (2nd column), and Wind (3rd column), in the $\left(\hat{\boldsymbol{x}}^{\prime}=\hat{\boldsymbol{n}} \times \hat{\boldsymbol{z}}, \hat{\boldsymbol{y}}^{\prime}=\hat{\boldsymbol{n}}, \hat{\boldsymbol{z}}^{\prime}=\hat{\boldsymbol{z}}\right)$ coordinates. The $\hat{\boldsymbol{z}}$ axis is the invariant axis and the normal $\hat{\boldsymbol{n}}$ is estimated for the bifurcated current sheet. The gray line denotes the regression line slope of \pm 1 . For all spacecraft the Walén slope is positive for the leading discontinuity and negative for trailing one.

Table 3. Summary of $\hat{z}, V_{0}, E_{0}, \varphi$, and $\alpha$ for ACE, Cluster, and Wind.

\begin{tabular}{lccccccccc}
\hline & & $\hat{z}[\mathrm{GSE}]$ & & \multicolumn{2}{c}{$\boldsymbol{V}_{0}[\mathrm{GSE}][\mathrm{km} / \mathrm{s}]$} & $E_{0}^{\mathrm{a}}$ & $\varphi^{\mathrm{b}}$ & $\alpha^{\mathrm{c}}$ \\
\hline ACE & 0.374 & -0.857 & -0.356 & -300.0 & -116.6 & -34.6 & -0.035 & 135 & 58 \\
Cluster* & 0.436 & -0.845 & -0.309 & -278.5 & -122.4 & -57.8 & -0.025 & 128 & 60 \\
Wind & 0.471 & -0.791 & -0.391 & -272.9 & -125.1 & -75.5 & -0.001 & 147 & 69 \\
\hline
\end{tabular}

*Combined data from $\mathrm{C} 1$ and $\mathrm{C} 3{ }^{\text {a }}$ Reconnection electric field, in $\mathrm{mV} / \mathrm{m}$ unit. ${ }^{\mathrm{b}}$ Magnetic shear angle, in degree unit. ${ }^{\mathrm{c}}$ The angle between the upstream magnetic field of the leading discontinuity and the X-line orientation, in degree unit.

data from Cluster, taken at the magnetopause, the $\hat{z}$ axis has also been estimated by optimizing the correlation coefficient between the magnetic fields measured by the other spacecraft and those predicted from the reconstruction map (Hasegawa et al., 2004). However, for the event studied here, the Cluster separation was much smaller than the size of the structure, a circumstance in which maximizing the correlation coefficient is not a reliable method for finding the $\hat{z}$ axis.

To determine the X-line motion and orientation for our events, we have divided the $\boldsymbol{V}_{0}$ vector into two parts: the motion $V_{n}$ along the normal $\hat{\boldsymbol{n}}$, and the motion $V_{t}$ along the transverse direction, $\hat{\boldsymbol{n}} \times \hat{\boldsymbol{z}}$. The value of $V_{t}$ and the orien- tation of the $\hat{z}$ axis are determined so as to optimize the constancy of $E_{z}$, i.e. by minimizing its fluctuations. Note that the $\hat{z}$ axis is constrained to be perpendicular to the normal $\hat{\boldsymbol{n}}$, that $V_{n}=\boldsymbol{V}_{\mathrm{HT}} \cdot \hat{\boldsymbol{n}}$, except as noted below, and that $E_{z}=-(\boldsymbol{V} \times \boldsymbol{B}) \cdot \hat{z}$, the axial convection electric field, is used to represent the total electric field. Table 3 summarizes the $\mathrm{X}$-line orientation, $\hat{z}$, its motion, $\boldsymbol{V}_{0}$, the average reconnection electric field, $E_{0}$, the magnetic shear angle, $\varphi$, and the angle, $\alpha$, between the upstream magnetic field of the leading discontinuity and the invariant axis $\hat{z}$.

For the ACE event, the $V_{n}$ value has been slightly adjusted from $\boldsymbol{V}_{\mathrm{HT}} \cdot \hat{\boldsymbol{n}}=-280.9 \mathrm{~km} / \mathrm{s}$ to $-278.5 \mathrm{~km} / \mathrm{s}$ in order 

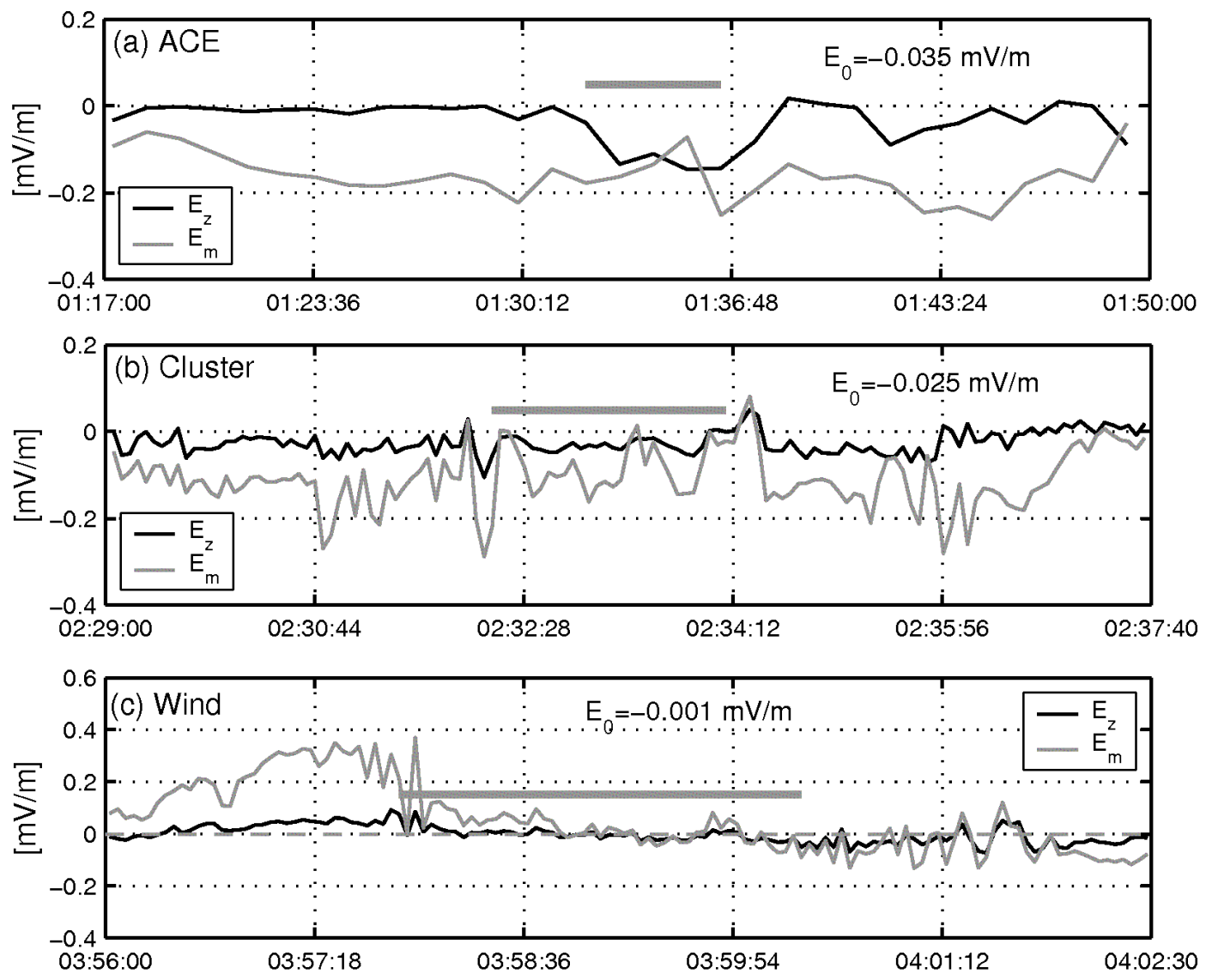

Fig. 5. Plots of the axial electric field $E_{z}$ (black) and the intermediate variance electric field $E_{m}$ (gray) for (a) ACE, (b) Cluster, and (c) Wind, in the reconstruction time interval. The axis $\hat{\boldsymbol{m}}=\hat{\boldsymbol{n}} \times \hat{\boldsymbol{z}}$. The gray bar denotes the reconnection exhaust region; the average $<E_{z}>=E_{0}$ over the entire reconstruction interval is shown by the dashed gray line.

to better maintain the constancy of $E_{z}$. By comparing the results from the traversals by ACE, Cluster and Wind, we find: (1) that the X-line orientation exhibits a small rotating motion toward the Sun-Earth line (an increase in the $x_{\mathrm{GSE}^{-}}$ component and a decrease in the $y_{\mathrm{GSE}}$-component) during the overall $\sim 2.42 \mathrm{~h}$ period between the ACE and Wind encounters; (2) that the X-line orientation for all three crossings is close to the bisector $(2 \alpha=\varphi)$ of the magnetic shear angle $\varphi$ (for Wind, Phan et al. (2006) chose $\hat{z}$ to precisely coincide with the bisector but used a somewhat different data interval for the determination); (3) that the X-line is moving antisunward, dawnward, and southward; (4) that the reconnection electric field, $E_{0}$, appears to decrease during the $\sim 2.42 \mathrm{~h}$ period and perhaps cease altogether near its end. Alternatively, the decrease could be a spatial effect, with the Wind event being located below one end of the reconnection line. Figure 5 shows the axial electric field, $E_{z}$, (black line) and the intermediate variance electric field, $E_{m}$, (gray line) for the three spacecraft. Note that the axis $\hat{\boldsymbol{m}} \equiv \hat{\boldsymbol{x}}^{\prime}=\hat{\boldsymbol{n}} \times \hat{\boldsymbol{z}}$ and that the maximum and minimum variances of the electric field are along the axis $\hat{\boldsymbol{n}}$ and $\hat{\boldsymbol{z}}$, respectively. As illustrated in Fig. 5, the electric field $E_{m}$ has large fluctuations compared to those of the axial electric field $E_{z}$. The latter is approximately constant for the Cluster and Wind events but not for the ACE event. Possible explanations for this behavior will be given in the discussion section. For the Wind encounter, our reconnection electric field is close to zero. It is much smaller in magnitude than the earlier result $(-0.03 \mathrm{mV} / \mathrm{m})$ inferred by Phan et al. (2006) from the change in the solar wind velocity component along the normal to the current sheet. The upstream and downstream regions they used are well outside our reconstruction interval. Our reconnection electric field is the average value over the entire reconstruction interval period. The field appears to have time-dependent behavior, as shown in Fig. 5c, including periods of positive and negative values. It is evident that there is a large uncertainty in our estimates of the reconnection electric field.

\section{Reconstruction method and results}

A full description of the MHD reconstruction theory and scheme has been given by Sonnerup and Teh (2008). Here we summarize it briefly. The method is based on 
integration of the ideal, single-fluid MHD equations with the assumptions of steady-state condition and two dimensions. The reconstruction is performed in the xy-plane (the reconstruction plane), i.e. in the plane perpendicular to the invariant axis, $\hat{z}$. In the reconstruction plane, the magnetic field $\boldsymbol{B}_{\perp}=\left[B_{x}(x, y), B_{y}(x, y)\right]$, flow field $\boldsymbol{V}_{\perp}=\left[V_{x}(x, y), V_{y}(x, y)\right]$, plasma density $\rho(x, y)$ and pressure $p(x, y)$, as well as the axial field $B_{z}(x, y)$, and axial flow $V_{z}(x, y)$, can all be generated in the reconstruction. In a steady state and two dimensions, Faraday's law requires the axial electric field to be constant, $E_{z}=E_{0}$, as mentioned already. The reconstruction is performed in the proper frame, moving with velocity $\boldsymbol{V}_{0}$, such that $\boldsymbol{V}_{0} \cdot \hat{z}=0$, relative to the observing spacecraft. It involves the integration of the MHD equations, using the quantities $[\boldsymbol{B}(x, 0), \boldsymbol{V}(x, 0), \rho(x, 0), p(x, 0)]$, measured along the spacecraft path (located at $y=0$ ), as spatial initial values. In the reconstruction plane, the magnetic field and flow field can be expressed by $\boldsymbol{B}_{\perp}=\nabla A \times \hat{\boldsymbol{z}}$ and $\boldsymbol{V}_{\perp}=(1 / \rho) \nabla \psi \times \hat{\boldsymbol{z}}$, respectively, where $A(x, y)$ is the vector potential and $\psi(x, y)$ is the compressible stream function. Field lines and streamlines are defined by $A(x, y)=$ const. and $\psi(x, y)=$ const., respectively, in the reconstruction plane. The initial values of $A(x, 0)$ and $\psi(x, 0)$ are obtained by integration of the measured $y$-components of field and flow:

$A(x, 0)=\int(\partial A / \partial x) d x=-\int B_{y}(x, 0) d x$,

$\psi(x, 0)=\int(\partial \psi / \partial x) d x=-\int \rho(x, 0) V_{y}(x, 0) d x$,

in which $d x=\left(\boldsymbol{V}_{0} \cdot \hat{\boldsymbol{x}}\right) d t$. In the integration process, the vector potential and stream function are advanced by use of the Taylor expansions

$A(x, y \pm \Delta y)=A(x, y) \pm \Delta y \partial A / \partial y+(1 / 2)(\Delta y)^{2} \partial^{2} A / \partial y^{2}(3)$

$\psi(x, y \pm \Delta y)=\psi(x, y) \pm \Delta y \partial \psi / \partial y+(1 / 2)(\Delta y)^{2} \partial^{2} \psi / \partial y^{2}(4)$

The quantities $\left[\rho, p, V_{z}, B_{z}\right]$ are advanced only to lowest order, e.g. $\rho(x, y \pm \Delta y)=\rho(x, y) \pm \Delta y \partial \rho / \partial y$. The unknown derivatives with respect to $y$, needed to advance the MHD system, can be arranged into the row vector

$\boldsymbol{X}=\left[\partial \rho / \partial y, \partial p / \partial y, \partial V_{z} / \partial y, \partial B_{z} / \partial y, \partial^{2} \psi / \partial y^{2}, \partial^{2} A / \partial y^{2}\right](5)$

This vector is governed by the matrix equation $\mathbf{M}_{0} \boldsymbol{X}^{T}=\boldsymbol{Y}_{0}^{T}$ where the matrix $\mathbf{M}_{0}$ and the vector $\boldsymbol{Y}_{0}^{T}$ contain only quantities and derivatives that are known at each step of the integration; their details are given in Sonnerup and Teh (2008). In the vector $\boldsymbol{Y}_{0}^{T}$, one of the terms is $\rho v_{x} d S / d \psi$, which comes from the conservation of entropy $S(\psi)$ along the streamlines. The function $S(\psi)$ and from it $d S / d \psi$ can be evaluated along the spacecraft path $(y=0)$ after $\psi(x, 0)$ has been obtained from Eq. (2).

\subsection{Reconstruction results from $\mathrm{ACE}$, Cluster, and wind}

The reconstruction intervals for ACE, Cluster, and Wind are enclosed by pairs of dashed purple lines in Fig. 2. The X-line orientation, $\hat{z}$, and its motion, $\boldsymbol{V}_{0}$, as well as the values of the reconnection electric field, $E_{0}$, are given in Table 3 for the three spacecraft. In the reconstruction coordinates, the axis $\hat{\boldsymbol{x}}=-\boldsymbol{V}_{0} /\left|\boldsymbol{V}_{0}\right|$ and the axis $\hat{\boldsymbol{y}}=\hat{\boldsymbol{z}} \times \hat{\boldsymbol{x}}$. To maintain the axial electric field $E_{z}=E_{0}$ throughout the reconstruction map, we nudge the axial electric field values at $y=0$ and also at each step of the integration by slightly adjusting the velocity components perpendicular to the magnetic field. However, there are some exceptions to this procedure. For example, as illustrated in Fig. 5a, the axial electric field for ACE has a large deviation from $E_{0}$, the average value of $E_{z}$, within the reconnection exhaust region (indicated by the grey bar). Thus, we could not preserve the axial electric field $E_{z}=E_{0}$ within that region without making large adjustments to the initial input values of the velocity. In such regions, we have omitted the nudging step. Figure 6 shows the plot of the entropy $S$ versus the stream function $\psi$, along with the fitting curve, for each of the spacecraft. To make the entropy values at $y=0$ fall exactly on the fitting curve, the measured pressure $p(x, 0)$ is somewhat adjusted. Pressure nudging is then applied after each step of the integration so as to keep the entropy $S(\psi)$ constant along the streamlines.

Figures 7, 8, and 9 show the reconstruction results for ACE, Cluster, and Wind, respectively. The format of the figures is the same: The first and second panels show the field line and streamline maps with the axial magnetic field $B_{z}$ and the plasma pressure $p$ in color; the third panel is an overlay of field lines in black and streamlines in white with the magnitude, $V_{\perp}$, of the transverse velocity in color. In the reconstruction maps, the left-hand side is toward Earth and the right-hand side toward Sun and the spacecraft moves from left to right across the map. In the first two panels of the reconstruction plots for the three spacecraft, one can see that the axial magnetic field, $B_{z}$, and the thermal pressure, $p$, appear to remain constant along field lines located outside the exhaust region, i.e. outside the region containing high thermal pressures and transverse speeds $V_{\perp}$. This constancy is expected because $B_{z}$ and $p$ are field-line invariants under the nearly magneto-hydrostatic conditions prevailing outside the exhaust. But, during the inflow into the exhaust region, inertial effects play an important role in the momentum equation. For ACE and Cluster, the axial magnetic field, $B_{z}$, in the exhaust region is relatively intense, compared to the Wind result. The similar behavior of $B_{z}$ in the ACE and Cluster maps is reasonable because their separation in the $\hat{\boldsymbol{y}}_{\mathrm{GSE}}$ direction is small. On the other hand, the Wind has a large $\hat{\boldsymbol{y}}_{\mathrm{GSE}}$ separation from ACE and Cluster. In the second and third panels of Figs. 7-9, the streamlines for ACE and Cluster illustrate the inflow from both sides of the exhaust wedge, while the inflow is not prominent and may even be absent at the earthwardfacing discontinuity in the Wind reconstruction map. For all 

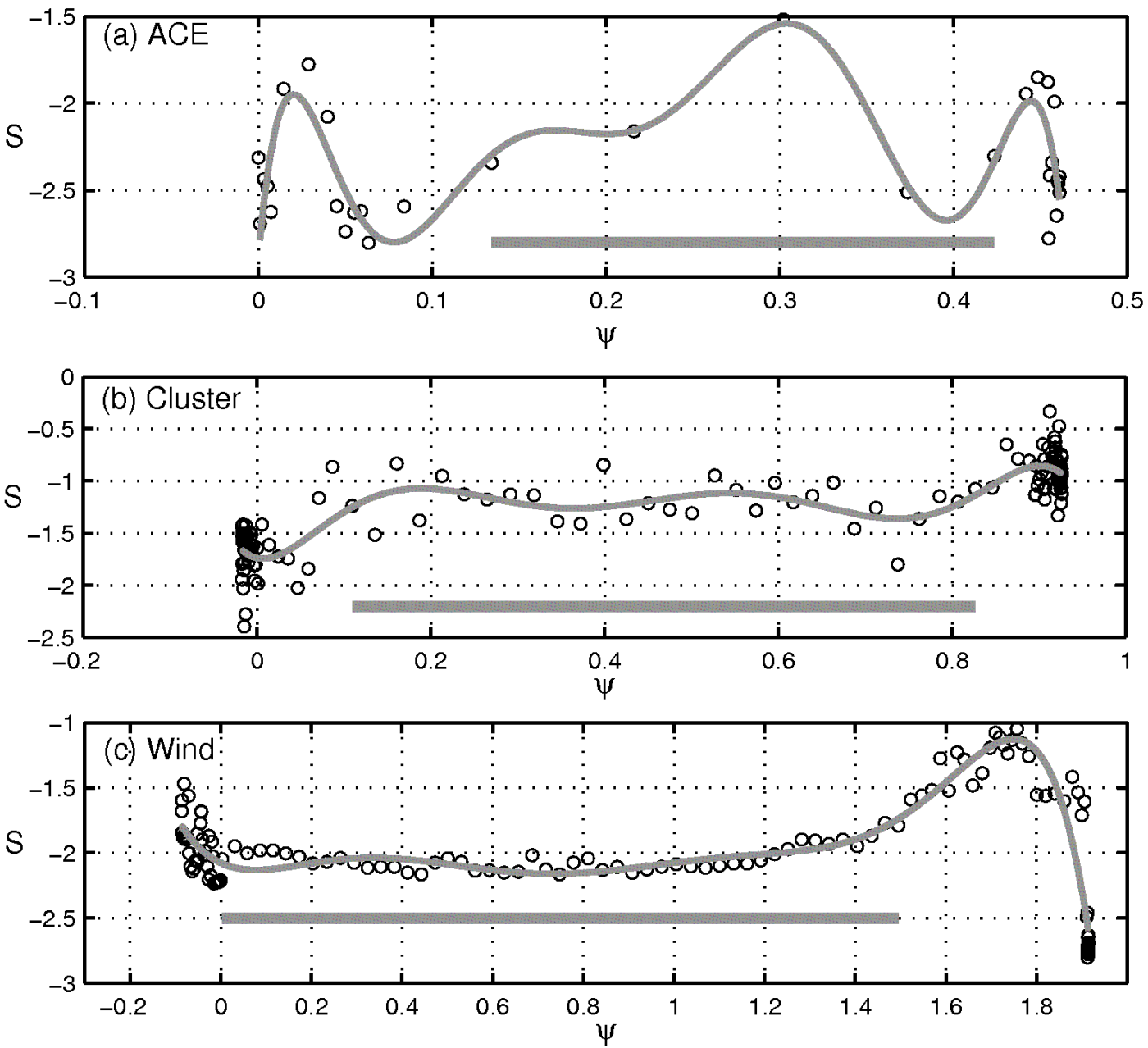

Fig. 6. Plots of entropy $S$ versus stream function $\psi$ along with the fitting curve (gray line) for (a) ACE, (b) Cluster, and (c) Wind. The grey bars denote the reconnection exhaust region. They correspond to the time intervals indicated by the corresponding grey bars in Fig. 5 .

three spacecraft, the X-line does not appear in the map but is located at a much larger positive $y$-value, as expected for the scenario of the event illustrated in Fig. 1. Note that the reconstruction $y$-axis is directed approximately along $+\hat{z}_{\mathrm{GSE}}$.

The magnetic field topology is similar for ACE and Cluster, while that for Wind is somewhat different. By examining the field line map in the first panel of Fig. 9 (Wind), we find that a magnetic island is embedded within the earthwardfacing discontinuity and is associated with a flow vortex, as shown in the second panel. Accordingly, the two exceptions encountered in the Wind event, namely the negative $B_{n}$ value and the negative flow value along the normal vector in the leading discontinuity, can be understood from the Wind reconstruction results: The negative $B_{n}$ value is caused by the existence of the magnetic island structure within the discontinuity and the negative inflow value results from the existence of the flow vortex. As shown in the third panel, there is a displacement between the center of the island and that of the vortex, indicating that the situation must be time depen- dent. In fact, the magnetic island and the vortex cannot be stationary in the reconstruction frame but both must be moving downward in the map; as a result, the island and vortex geometry in Fig. 9 is time-aliased.

The reconstruction maps from the three spacecraft are consistent with the finding by Phan et al. (2006) that reconnection occurred and persisted for more than $2.4 \mathrm{~h}$ : (1) The magnetic field topology characteristic of reconnection is present in the reconstruction maps from all three spacecraft; (2) Enhanced plasma flow is present in the exhaust region, as expected.

\section{Summary and discussion}

We have, for the first time, reconstructed 2-D magnetic field and flow field configurations showing portions of a largescale reconnection exhaust region in the solar wind, observed first by ACE, followed by Cluster, and finally by Wind, on 

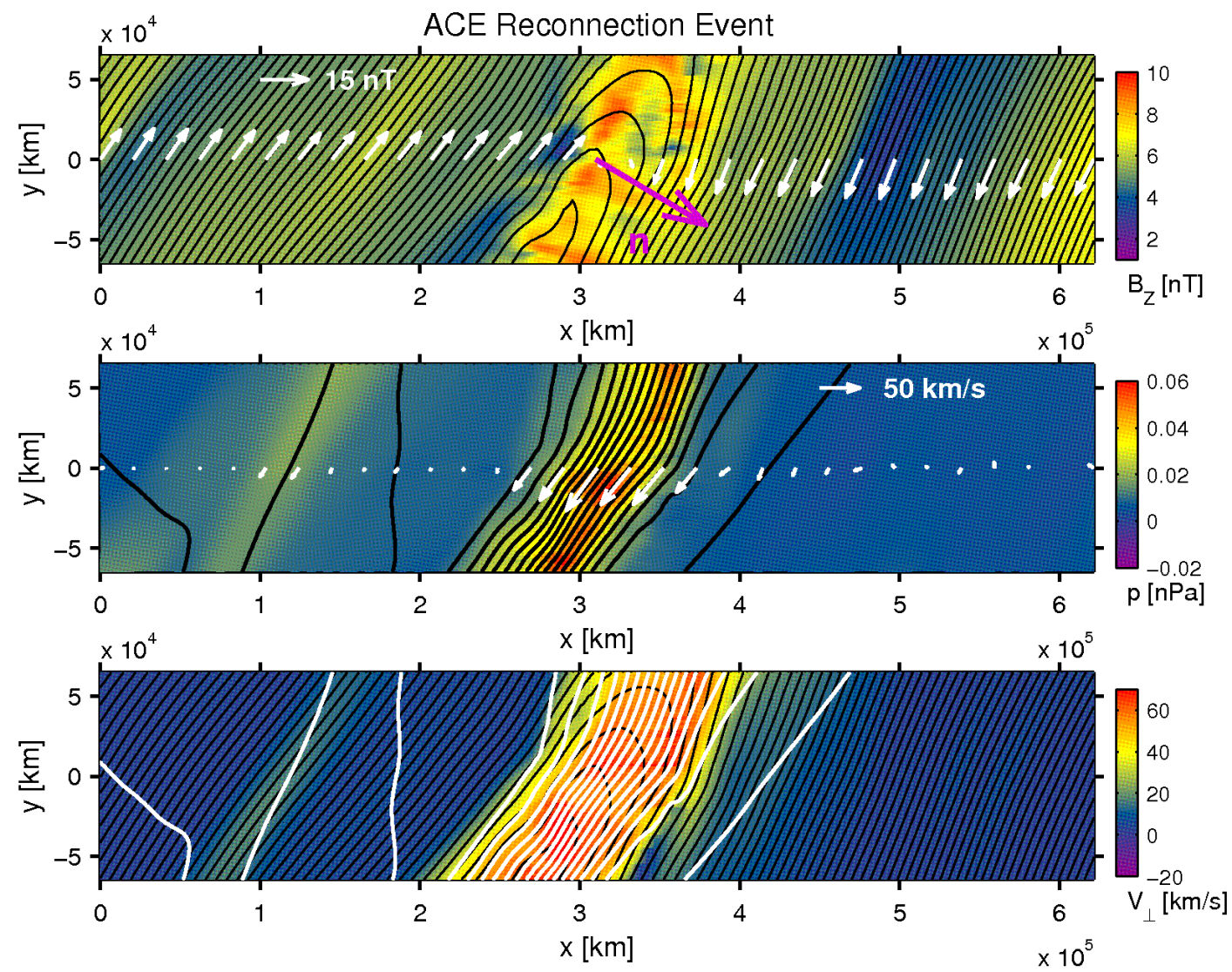

Fig. 7. MHD reconstruction maps of the bifurcated current sheet seen by ACE on 2 February 2002, during the interval of 01:17:00-01:50:00 UT. The top panel shows the magnetic field lines map with $B_{z}$ in color; white arrows are the measured magnetic fields projected onto the reconstruction plane and the violet arrow is the sunward-pointing normal vector for the combined bifurcated structure. The second panel shows the streamlines map with the pressure $p$ in color; white arrows are the measured velocities in the X-line frame, projected onto the reconstruction plane. The third panel is an overlay of field lines (black) and streamlines (white), with the magnitude, $V_{\perp}$, of the transverse velocity in color. Earth is to the left; Sun is to the right; north is up.

2 February 2002. The event was first identified by Phan et al. (2006) as a significant example of a quasi-steady, largescale reconnection configuration in the solar wind. The bifurcated current sheet containing the reconnection exhaust consists of two discontinuities, both of which contain elements of intermediate-mode and slow-mode behavior. The two elements do not have the spatial separation predicted by MHD theory; they seem to be co-located. The coplanarity condition, i.e. the coplanarity of the normal vector with the upstream and downstream field vectors, as required for all shocks, is not satisfied for any of the six discontinuities (see Fig. 3). The trailing discontinuity seen by Wind almost satisfies the condition and has the qualitative behavior expected for a slow-mode shock. However, the MHD jump conditions are not well satisfied and the magnetic field behavior within the shock layer remains unexplained. The leading discontinuity seen by Wind has mostly intermediate-mode characteristics; it could be a rotational discontinuity, except that the field magnitude changes across it and the plasma inflow speed, as seen in the HT frame, is significantly sub-Alfvénic. The sub-Alfvénic inflow, along with the fact that the field rotation angle is significantly less than $180^{\circ}$, also prevents the interpretation as an intermediate-mode shock. Because the plasma $\beta$ is small, the sub-Alfvénic inflow cannot be the result of our neglect of the pressure anisotropy factor in the Alfvén speed. The uncertainty in the Walén slope $(0.7 \pm 0.02)$ is also too small to provide an explanation.

As shown in Fig. 4, the observations by all three spacecraft have a positive Walén slope and a reversal of the magnetic field component perpendicular to the invariant axis $\hat{z}$ in the leading discontinuity and a negative Walén slope with no such field reversal in the trailing discontinuity. The different magnetic field reversal behavior across the leading and trailing discontinuities is also illustrated in Fig. 3. It is a consequence of differences in the plasma and field properties in the regions ahead of and behind the bifurcated current sheet. The sign reversal of the Walén slope is consistent with the geometry in Fig. 1. Together, they indicate inflow into the 

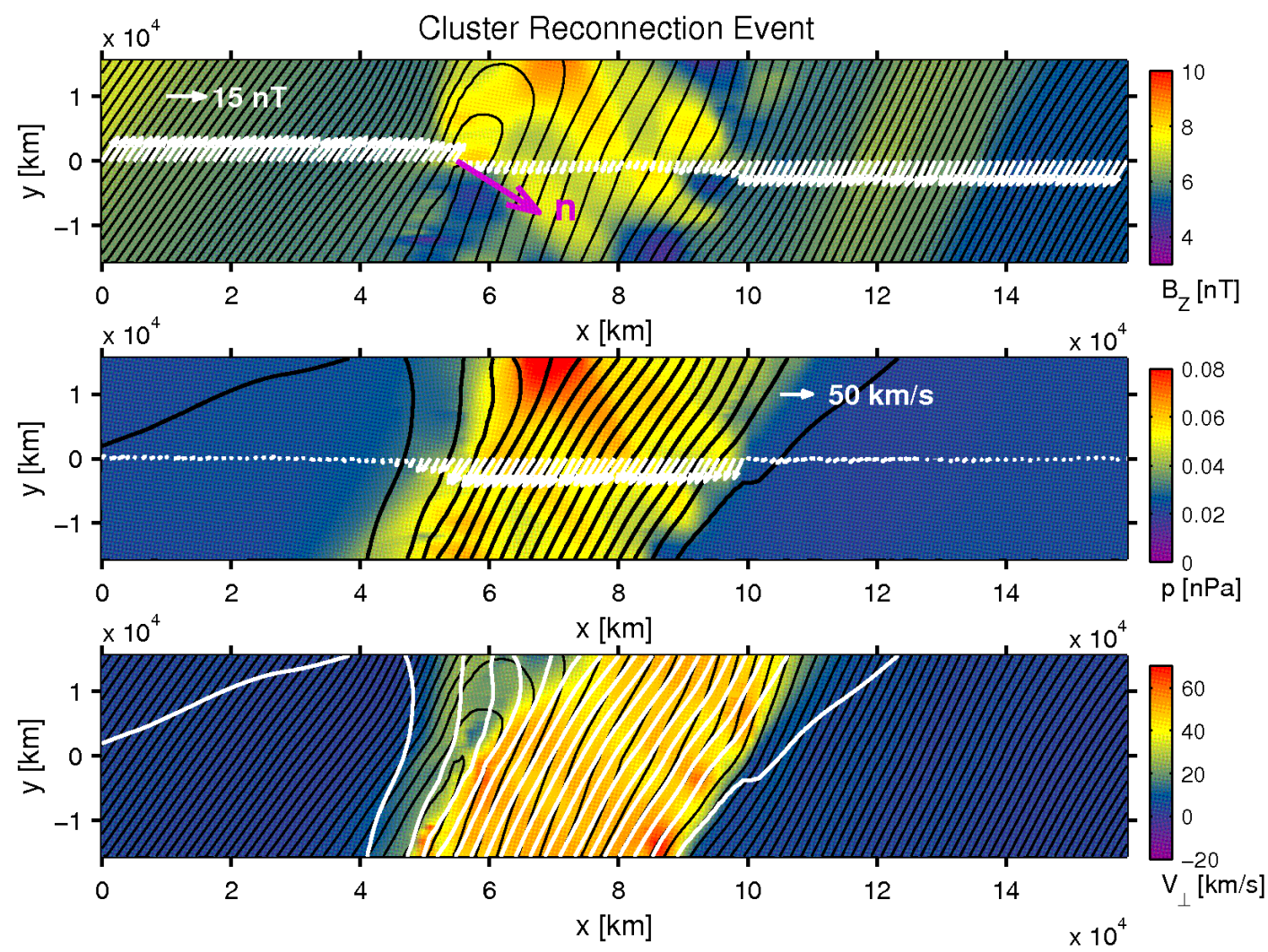

Fig. 8. MHD reconstruction maps of the bifurcated current sheet seen by Cluster on 2 February 2002, during the interval of 02:29:0002:37:40 UT. Format is the same as in Fig. 7 but note that the coordinate scales have changed.

exhaust region from both sides and a normal field component $B_{n}$ that is positive at both the leading and the trailing discontinuities. With the exception of the leading discontinuity seen by Wind, this behavior of the normal plasma inflow, $v_{n}$, and field $B_{n}$ is also what our chosen normal vectors give. The exception for the leading Wind discontinuity is caused by the vortex and associated magnetic island shown in the reconstruction map (Fig. 9). This feature leads to a negative value of both $v_{n}$ and $B_{n}$ at the location of the spacecraft trajectory $(y=0)$.

Note that the vortex and the magnetic island in Fig. 9 do not precisely coincide. This is a clear indication that these structures are not stationary in the reconstruction (X-line) frame. The expectation is that they must be moving downward (toward negative $y$-values). To confirm this expectation, we have performed the MHD reconstruction in a frame that moves with the local HT velocity. The result is shown in Fig. 10. We see that the flow field is now well aligned with the magnetic field. The downward speed of the island, as seen in the reconstruction plane anchored in the X-line, can be estimated by subtracting the X-line velocity from the HT velocity: the result is $\sim 45 \mathrm{~km} / \mathrm{s}$, which is $\sim 75 \%$ of the exhaust speed in the X-line frame.
With the analysis results in Table 3 for the X-line orientation and motion, as well as the reconnection electric field, we have demonstrated: (1) that the X-line orientation is close to the bisector of the magnetic shear angle, that it points approximately dawnward, and that it exhibits a slow rotating motion toward the Sun-Earth line; (2) that the X-line motion is earthward, dawnward, and southward; (3) that the reconnection electric field appears to decrease gradually and perhaps vanish altogether at the time (or location) of the Wind encounter. The wedge width estimated from the reconstruction map is $\sim 10 \times 10^{4} \mathrm{~km}, 6 \times 10^{4} \mathrm{~km}$, and $4 \times 10^{4} \mathrm{~km}$ for ACE, Wind, and Cluster, respectively. The $\mathrm{X}$-line is persistently located north of the three spacecraft. By assuming, as an extreme case, that it was exactly encountered by the Wind spacecraft (whereas it was in fact substantially north of Wind), we can use its average velocity to obtain a lower bound on its distance from ACE in the reconstruction plane at the time of the ACE encounter. As shown by the gray bar in Fig. 1, the resulting lower bound for the distance along the wedge between the X-line and the ACE spacecraft is about $156 R_{E}$. Using this distance and the ACE and Wind wedge widths from the reconstructions, we can thus obtain a distance of $234 R_{E}$ between the X-line and the ACE spacecraft as shown in Fig. 1. This distance is substantially larger than 

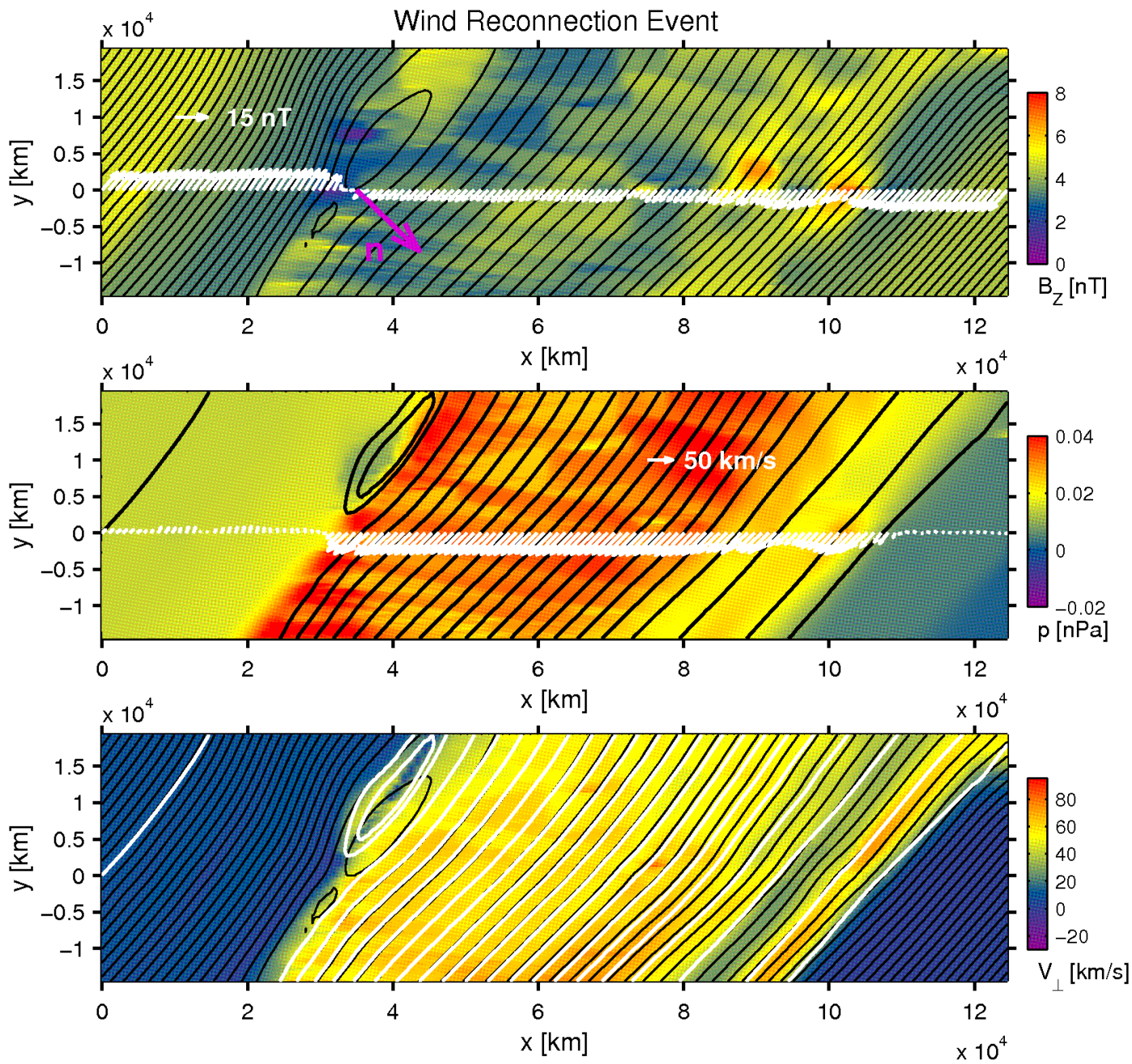

Fig. 9. MHD reconstruction maps of the bifurcated current sheet seen by Wind on 2 February 2002, during the interval of 03:56:0004:02:30 UT. Format is the same as in Fig. 7 but again the coordinate scales are different from those in Figs. 7 and 8.

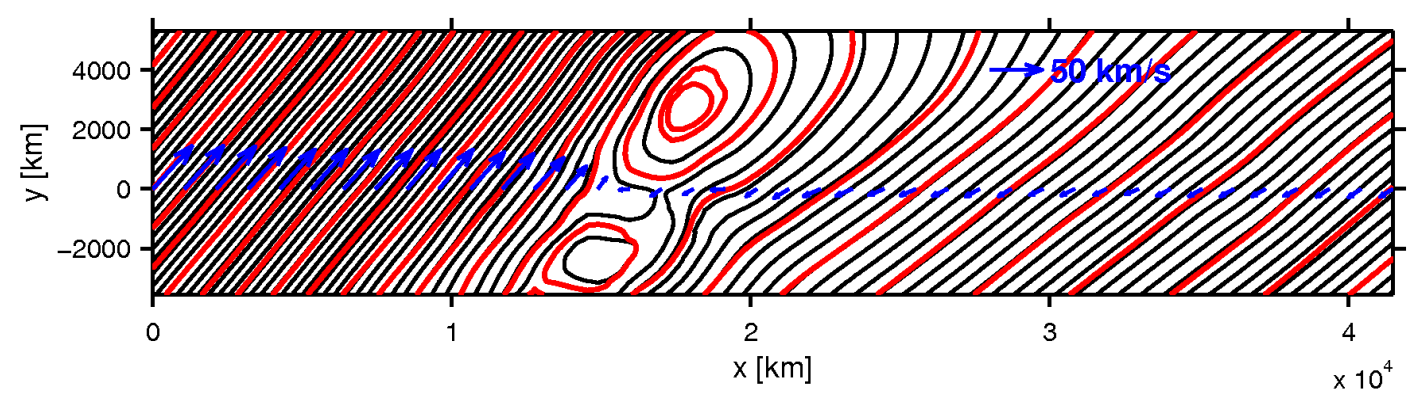

Fig. 10. Overlay of field lines (black) and streamlines (red) for the leading discontinuity seen by Wind in the interval of 03:57:0003:59:00 UT. The results were obtained from MHD reconstruction in the HT frame. Blue arrows are the measured velocities, transformed to the HT frame, and projected onto the reconstruction plane.

the predictions for ACE using the wedge angle $\theta$ in Table 1 together with the width of the exhaust region. Likely explanations for this discrepancy are uncertainties in the $\theta$ values and variations in $\theta$ along the exhaust jet, caused by a timedependent reconnection rate.
In the Cluster reconstruction results, the correlation coefficient (cc) between the magnetic fields measured by $\mathrm{C} 1$, $\mathrm{C} 2$, and $\mathrm{C} 4$ and those predicted from the map based on the combined data from $\mathrm{C} 1$ and $\mathrm{C} 3$ is approximately 0.99 . The correlation coefficients between the velocity, density, and 

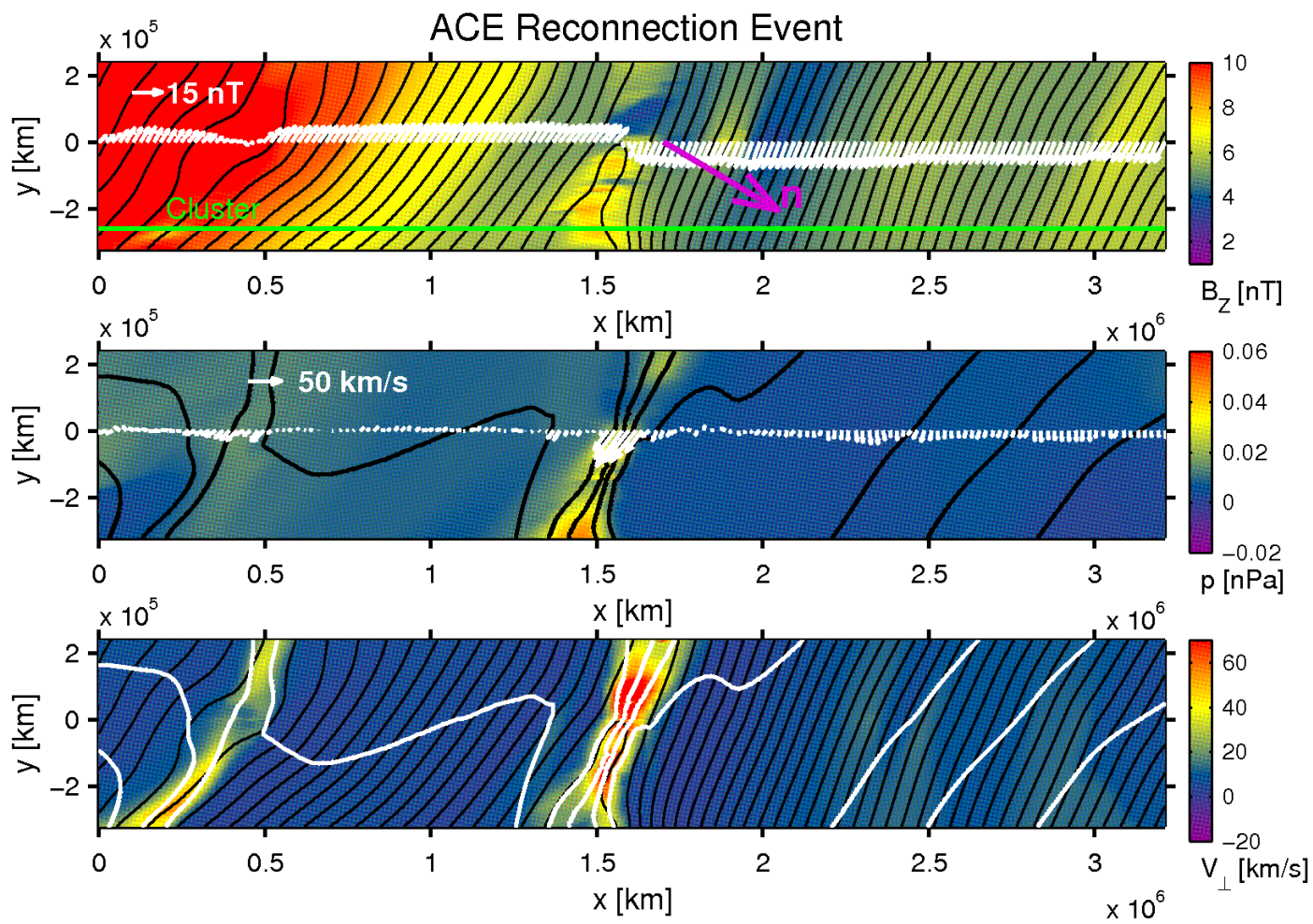

Fig. 11. MHD reconstruction maps of the bifurcated current sheet seen by ACE on 2 February 2002, during the interval of 00:12:1202:57:32 UT. Format is the same as in Fig. 7 but scales are larger. The green line in the first panel is the Cluster trajectory.

pressure measured by $\mathrm{C} 1$ and the corresponding predictions are $\sim 0.99, \sim 0.98$, and $\sim 0.90$, respectively. The high correlation indicates that the structure is essentially time independent as it blows past the Cluster spacecraft. As illustrated in Fig. 1, the spacecraft separations between ACE and Cluster and between $\mathrm{ACE}$ and Wind are $\sim 31 R_{E}$ and $\sim 110 R_{E}$, respectively, in the reconstruction $\hat{\boldsymbol{y}}$ direction. As a result of growing numerical errors, the MHD reconstruction results from ACE in Fig. 7 cannot be extended to the Cluster and Wind locations. However, by use of a longer data interval they can reach Cluster. Figure 11 shows the ACE reconstruction maps with the Cluster trajectory (green line) for the time interval 00:12:12-02:57:32 UT, using the same format as in Fig. 7. Comparison of the Cluster observations with the predictions from $\mathrm{ACE}$ gives $\mathrm{cc}=0.96$ for the magnetic field. We have also done magneto-hydrostatic GS reconstruction for ACE, giving again $\mathrm{cc}=0.96$ for the magnetic field at Cluster. Figure 12 shows the comparison between the magnetic fields measured by Cluster (black) and the corresponding values predicted by the MHD reconstruction (red) and the magneto-hydrostatic GS reconstruction (blue), based on data from ACE. Although the reconstruction cannot recover the abruptness of the change in $B_{x}$ and $B_{y}$ seen by Cluster, Fig. 12 confirms that, in an overall sense, ACE and Cluster were observing the same wedge-like exhaust region.
The plots in Fig. 6 show that the entropy is highly variable in the reconstruction interval and is in fact not the same in the solar wind on its two sides. Within the exhaust region (denoted by the grey bar), the entropy is approximately constant for Cluster and Wind but not for ACE. Peaks, associated with the discontinuities, are seen near the two ends of the $\psi$-range, a feature that is not readily explainable in our MHD model. The entropy jumps across the discontinuities also appear inconsistent. It must be remembered that the plasma near the center of the exhaust region crossed the entropy-producing discontinuities at a large distance (towards the X-line) away from the observing spacecraft and at a substantially earlier time. Qualitatively, the entropy variations in Fig. 6 could perhaps be thought of as a combined record of spatial variations with distance from the $\mathrm{X}$-line and temporal variations.

In the reconstructed maps, the entropy is conserved along streamlines. This behavior is appropriate for almost all of the streamlines shown, even those within the exhaust region, because they do not cross the entropy-producing shock-like regions within the domain depicted in the maps. For this reason, the assumption of constant entropy along streamlines is unlikely to compromise the quality of the maps in a significant way.

As shown in Fig. 5, the axial electric field is approximately constant for Cluster and Wind but not for ACE. For ACE, the axial electric field is smaller outside the exhaust wedge 
than inside it. A possible explanation is that the two discontinuities of the bifurcated current sheet were expanding away from each other which, according to Faraday's law, would lead to an enhanced negative axial electric field in the exhaust region, as is indeed seen in the top panel of Fig. 5. This interpretation is in qualitative agreement with the MVAB result that the angle $\theta$ between the two discontinuities is larger for ACE than for Wind (see Table 1). For Wind, we see that the axial electric field is positive prior to the arrival of the leading (earthward-facing) discontinuity. This behavior is, we argue, a consequence of the magnetic island which causes a negative value of $B_{n}$ during the traversal of the discontinuity as the island moves toward negative $y$-values. This motion will give rise to a positive axial electric field. We also see that the axial electric field seen by Wind is very small in the exhaust region. To explain this result, we conjecture that the two discontinuities of the bifurcated current sheet may have been collapsing toward each other, as a result of a shut-down of the reconnection process. This conjecture is consistent with the change across the current sheet of the solar wind velocity component along $\hat{\boldsymbol{n}}$, interpreted by Phan et al. (2006) as inflow into the exhaust region and forming the basis for their estimate of the reconnection electric field during the Wind crossing. It is also qualitatively supported by additional observations: (1) the angle $\theta$ is smaller for Wind than for ACE; (2) the combined ion and magnetic pressure seen by Wind is weaker within the exhaust region than outside it, as illustrated in Fig. 2; (3) the inflow speed (blue triangles in Fig. 4) into the exhaust region is very small on average. Note that the reconnection jet would not disappear immediately upon the cessation of reconnection: All the exhaust plasma between the X-point and the observation point first needs to be drained.

With regard to item (2) above, we stress that the total pressure shown in Fig. 2 (bottom panels) does not include the electron pressure which is usually significant in the solar wind. In fact, the total pressure depression, seen by Wind in the exhaust jet (Fig. 2, bottom right panel), is much too deep to be explained solely in terms of collapse of the bifurcated current sheet. But our point remains that what is interpreted in a steady-state model as inflow into the exhaust region from its two sides could instead be accounted for an ongoing collapse of that region.

According to the traditional MHD reconnection model, the dimensionless reconnection rate, expressed as $\sim B_{n} / B_{\perp}$, should be approximately equal to half of the wedge angle $\theta$. However, we find that the angles resulting from $B_{n} / B_{\perp}$ are larger than the $\theta$ values given in Table 1 . We argue that this discrepancy is caused principally by the uncertainty of the normal vector estimation. The reconnection rate can also be expressed as $V_{n} / V_{A}=\left(E_{0} / B_{\perp}^{2}\right) \sqrt{\mu_{0} \rho}=\theta / 2$. Using the average reconnection electric field over the three spacecraft in this formula, we find the wedge angle $\theta$ predicted from this formula to be about $2.5^{\circ}$, which is considerably smaller than the values given in Table 1 .

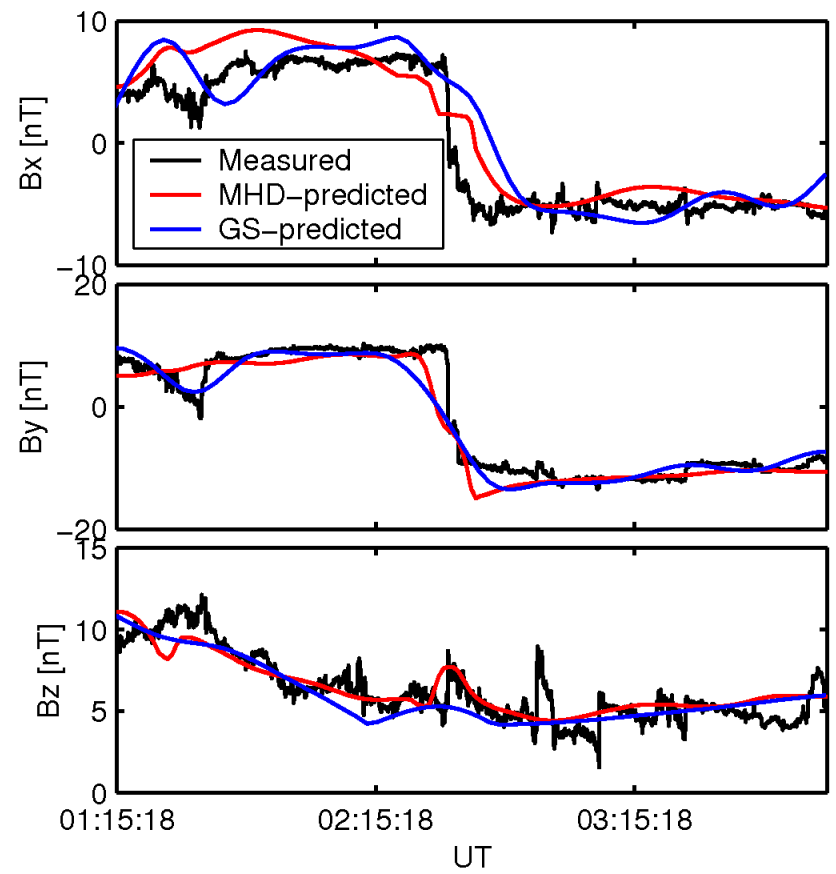

Fig. 12. A time series plot of the magnetic fields measured by Cluster (black) and the corresponding values predicted by the MHD reconstruction (red) and the magneto-hydrostatic GS reconstruction (blue) from ACE. Both reconstructions give a correlation coefficient of 0.96 . The $[x, y, z]$ coordinates are the reconstruction axes, where the $\mathrm{z}$-axis is the invariant axis.

In conclusion, with the help of the MHD reconstruction method we have recovered 2-D magnetic field and flow field configurations within a large-scale reconnection event in the solar wind, as seen by three widely separated spacecraft. Although there are some differences in details, our overall results are in agreement with those reported by Phan et al. (2006) for this event. In particular, we find: (1) that the average reconnection electric field for the three spacecraft is very small, typically $\sim-0.02 \mathrm{mV} / \mathrm{m}$; (2) that there is strong evidence of plasma inflow into the exhaust wedge from its two sides.

\section{Appendix A}

We present a method for using data from only three spacecraft to estimate the orientation and average motion of a current sheet. The method involves using the constraint that $\hat{\boldsymbol{n}}$ be perpendicular to the maximum variance direction, as suggested by Sonnerup et al. (2008). Let $\hat{\boldsymbol{x}}_{1}, \hat{\boldsymbol{x}}_{2}$, and $\hat{\boldsymbol{x}}_{3}$ be, respectively, the maximum, intermediate, and minimum variance directions, estimated from MVAB applied to the combined set of magnetic field vectors from the three spacecraft. Suppose $\hat{\boldsymbol{n}} \cdot \hat{\boldsymbol{x}}_{1}=0$, so that $\hat{\boldsymbol{n}}=n_{2} \hat{\boldsymbol{x}}_{2}+n_{3} \hat{\boldsymbol{x}}_{3}$, where $n_{2}$ and $n_{3}$ are to be determined. Note that $n_{2}^{2}+n_{3}^{2}=1$. Using the relative position vectors, $\boldsymbol{r}$, and the propagation times, 
$t$, of the current sheet from ACE to Cluster and from ACE to Wind, one then obtains $\boldsymbol{r}_{1} \cdot \hat{\boldsymbol{n}}=\left(\boldsymbol{r}_{1} \cdot \hat{\boldsymbol{x}}_{2}\right) n_{2}+\left(\boldsymbol{r}_{1} \cdot \hat{\boldsymbol{x}}_{3}\right) n_{3}=V_{n} t_{1}$ and $\boldsymbol{r}_{2} \cdot \hat{\boldsymbol{n}}=\left(\boldsymbol{r}_{2} \cdot \hat{\boldsymbol{x}}_{2}\right) n_{2}+\left(\boldsymbol{r}_{2} \cdot \hat{\boldsymbol{x}}_{3}\right) n_{3}=V_{n} t_{2}$, where the subscript 1 (2) is for ACE-Cluster (ACE-Wind) and $V_{n}$ is the average speed of the current sheet along the normal vector. In matrix form, these equations give

$$
\left[\begin{array}{ll}
\boldsymbol{r}_{1} \cdot \hat{\boldsymbol{x}}_{2} & \boldsymbol{r}_{1} \cdot \hat{\boldsymbol{x}}_{3} \\
\boldsymbol{r}_{2} \cdot \hat{\boldsymbol{x}}_{2} & \boldsymbol{r}_{2} \cdot \hat{\boldsymbol{x}}_{3}
\end{array}\right]\left[\begin{array}{l}
m_{2} \\
m_{3}
\end{array}\right]=\left[\begin{array}{l}
t_{1} \\
t_{2}
\end{array}\right],
$$

where $m_{2}=n_{2} / V_{n}$ and $m_{3}=n_{3} / V_{n}$. After solving Eq. (A1) for $m_{2}$ and $m_{3}$, the velocity $V_{n}$ can be calculated from $m_{2}^{2}+m_{3}^{2}=1 / V_{n}^{2}$, and finally $n_{2}=V_{n} m_{2}$ and $n_{3}=V_{n} m_{3}$ can be obtained.

Acknowledgements. The research by W.-L. T. and B. U. Ö. S. was supported by the National Aeronautics and Space Administration (NASA) under Cluster Theory Guest Investigator Grant NNG-05GG26G to Dartmouth College. Q. H. was supported by NASA Grants NNG-04GF47G and NNG-06GD41G and C. J. F. by NASA Wind Grant NNX-08AD11G and NASA Cluster Specific Theory and Modeling Grant NNG-05G158G to UNH. We thank the CDAWeb and Cluster Active Archive (CAA) for providing the data. We also thank T. D. Phan for helpful comments on the manuscript.

Topical Editor I. A. Daglis thanks I. Richardson and another anonymous referee for their help in evaluating this paper.

\section{References}

Gosling, J. T., Skoug, R. M., McComas, D. J., and Smith, C. W.: Direct evidence for magnetic reconnection in the solar wind near $1 \mathrm{AU}$, J. Geophys. Res., 110, A01107, doi:10.1029/2004JA010809, 2005.

Gosling, J. T., Eriksson, S., Blush, L. M., Phan, T. D., Luhmann, J. G., McComas, D. J., Skoug, R. M., Acuna, M. H., Russell, C. T., and Simunac, K. D.: Five spacecraft observations of oppositely directed exhaust jets from a magnetic reconnection X-line extending $>4.26 \times 10^{6} \mathrm{~km}$ in the solar wind at $1 \mathrm{AU}$, Geophys. Res. Lett., 34, L20108, doi:10.1029/2007GL031492, 2007.

Harvey, C. C.: Spatial gradients and the volumetric tensor, in: Analysis Methods for Multi-Spacecraft Data, edited by: Paschmann, G. and Daly, P. W., ISSI SR-001, Chapter 12, International Space Science Institute, Bern, Switzerland, 1998.

Hasegawa, H., Sonnerup, B. U. Ö., Dunlop, M. W., Balogh, A., Haaland, S. E., Klecker, B., Paschmann, G., Lavraud, B., Dandouras, I., and Rème, H.: Reconstruction of two-dimensional magnetopause structures from Cluster observations: verification of method, Ann. Geophys., 22, 1251-1266, 2004, http://www.ann-geophys.net/22/1251/2004/.
Hasegawa, H., Sonnerup, B. U. Ö., Klecker, B., Paschmann, G., Dunlop, M. W., and Rème, H.: Optimal reconstruction of magnetopause structures from Cluster data, Ann. Geophys., 23, $973-$ 982, 2005, http://www.ann-geophys.net/23/973/2005/.

Hau, L.-N. and Sonnerup, B. U. Ö.: Two-dimensional coherent structures in the magnetopause: Recovery of static equilibria from single-spacecraft data, J. Geophys. Res., 104, 6899-6917, 1999.

Hu, Q. and Sonnerup, B. U. Ö.: Reconstruction of two-dimensional structures in the magnetopause: Method improvements, J. Geophys. Res., 108(A1), 1011, doi:10.1029/2002JA009323, 2003.

Petschek, H. E. and Thorne, R. M.: The existence of intermediate waves in neutral sheets, Astrophys. J., 147(3), 1157-1163, 1967.

Phan, T. D., Gosling, J. T., Davis, M. S., Skoug, R. M., Øieroset, M., Lin, R. P., Lepping, R. P., McComas, D. J., Smith, C. W., Rème, H., and Balogh, A.: A magnetic X-line extending more than 390 Earth radii in the solar wind, Nature, 439, 175-178, 2006.

Sonnerup, B. U. Ö. and Cahill Jr., L. J.: Magnetopause structure and attitude from Explorer 12 observations, J. Geophys. Res., 72, 171-183, 1967.

Sonnerup, B. U. Ö. and Guo, M.: Magnetopause transects, Geophys. Res. Lett., 23, 3679-3682, 1996.

Sonnerup, B. U. Ö. and Hasegawa, H.: Orientation and motion of two-dimensional structures in a space plasma, J. Geophys. Res., 110, A06208, doi:10.1029/2004JA010853, 2005.

Sonnerup, B. U. Ö., Hasegawa, H., Teh, W.-L., and Hau, L.-N.: Grad-Shafranov reconstruction: An overview, J. Geophys. Res. 111, A09204, doi:10.1029/2006JA0117, 2006.

Sonnerup, B. U. Ö. and Teh, W.-L.: Reconstruction of twodimensional coherent MHD structures in a space plasma, J. Geophys. Res., 113, A05202, doi:10.1029/2007JA012718, 2008.

Sonnerup, B. U. Ö., Haaland, S. E., and Paschmann, G.: Discontinuity orientation, motion, and thickness, in: Multi-Spacecraft Analysis Methods Revisited, edited by: Paschmann, G. and Daly, P. W., ISSI SR-008, Chapter 1, International Space Science Institute, Bern, Switzerland, 2008.

Teh, W.-L., Sonnerup, B. U. Ö., and Hau, L.-N.: Grad-Shafranov reconstruction with field-aligned flow: First results, Geophys. Res. Lett., 34, L05109, doi:10.1029/2006GL028802, 2007.

Teh, W.-L. and Sonnerup, B. U. Ö.: First results from ideal 2-D MHD reconstruction: magnetopause reconnection event seen by Cluster, Ann. Geophys., 26, 2673-2684, 2008, http://www.ann-geophys.net/26/2673/2008/. 